\title{
Storage of Nuclear Excitation Energy through Magnetic Switching
}

\author{
Yu. V. Shvyd'ko, ${ }^{1}$ T. Hertrich, ${ }^{2}$ U. van Bürck, ${ }^{2}$ E. Gerdau, ${ }^{1}$ O. Leupold,${ }^{1}$ J. Metge, ${ }^{1}$ H. D. Rüter, ${ }^{1}$ S. Schwendy, ${ }^{1}$ \\ G. V. Smirnov, ${ }^{3}$ W. Potzel, ${ }^{2}$ and P. Schindelmann ${ }^{2}$ \\ ${ }^{1}$ II. Institut für Experimentalphysik, Universität Hamburg, D-22761 Hamburg, Germany \\ ${ }^{2}$ Physik-Department E15, Technische Universität München, D-85748 Garching, Germany \\ ${ }^{3} R R C$, "Kurchatov Institute", SU-1123182 Moscow, Russia
}

(Received 8 May 1996)

\begin{abstract}
The decay rate of ${ }^{57} \mathrm{Fe}$ nuclei in an ${ }^{57} \mathrm{FeBO}_{3}$ crystal excited by $14.4 \mathrm{keV}$ synchrotron radiation pulses was controlled by switching the direction of the crystal magnetization. Abrupt switching some nanoseconds after excitation suppresses the coherent nuclear decay. Switching back at later times restores it, starting with an intense radiation spike. The enhanced delayed reemission is due to the release of the energy stored during the period of suppression. Suppression and restoration originate from drastic changes of the nuclear states and of the interference within the nuclear transitions. [S0031-9007(96)01296-3]
\end{abstract}

PACS numbers: 76.80.+y, 42.25.-p, 42.50.Md, 78.70.Ck

The feasibility to change the radiation of a coherently excited nuclear ensemble by switching abruptly the directions of the magnetic hyperfine fields was demonstrated in nuclear Bragg scattering of synchrotron radiation [1]. In the present experiment this technique was used to switch the coherent decay channel of a nuclear excitation off and on. The central idea was to study the possibility of storing excitation energy by closing the coherent decay and releasing the stored energy later.

Nuclear resonant excitation produced by a photon may decay coherently and incoherently. In a large ensemble of nuclei, the spatially coherent decay channels into the Bragg directions and into the forward direction are dominant ([2,3]; for a review see [4]). They are also considerably speeded up with respect to the incoherent decay channels, which have the natural lifetime $\tau_{0}$. Thus a suppression of the coherent decay channels should result in an essential slowing down of the total decay and in a storage of nuclear excitation energy.

The properties of coherent excitations in resonant systems and, in particular, the question, to which extent such excitations can be manipulated, are of general interest in physics [5]. Coherent effects strongly affect the decay rates of classical systems as well as of quantum systems [4]. Such effects have been observed in optics for atomic [6], excitonic [7] resonances, and recently even for isolated two atom systems [8] where the coherent decay was manipulated by variation of the interatomic distance. For our experiment a nuclear resonance was employed, because nuclear systems constitute ideal model oscillators with coherent and incoherent decay channels of suitable time scales, which moreover can be very fast manipulated by magnetic interaction. A scattering geometry with only one spatially coherent channel, namely, forward scattering, was used.

Time spectra of the nuclear forward scattering were studied in a ${ }^{57} \mathrm{FeBO}_{3}$ single crystal after the excitation of the $14.4 \mathrm{keV}{ }^{57} \mathrm{Fe}$ nuclear resonance $\left(\tau_{0}=141.1 \mathrm{~ns}\right)$ by synchrotron radiation pulses. The experiments were performed at the bending magnet beam line F4 at HASYLAB (Hamburg) [9]. The synchrotron radiation was monochromatized to $6 \mathrm{meV}$ by using a high resolution monochromator [10] and directed to the ${ }^{57} \mathrm{FeBO}_{3}$ crystal plate perpendicular to its surface (111). The scattering geometry is depicted in Fig. $1 . \mathrm{FeBO}_{3}$ is a canted anitferromagnet with a plane of easy magnetization parallel to the (111) planes. $\mathrm{FeBO}_{3}$ was chosen because of its excellent magnetic switching properties [1]. The crystal was $23 \pm 3 \mu \mathrm{m}$ thick and enriched in ${ }^{57} \mathrm{Fe}$ to $95 \%$. A constant magnetic field $H_{c}=20 \mathrm{G}$ induced a magnetization parallel to the crystal plane surface and aligned the magnetic hyperfine fields $\boldsymbol{B}_{0}^{(n)}$ at the nuclei of both sites

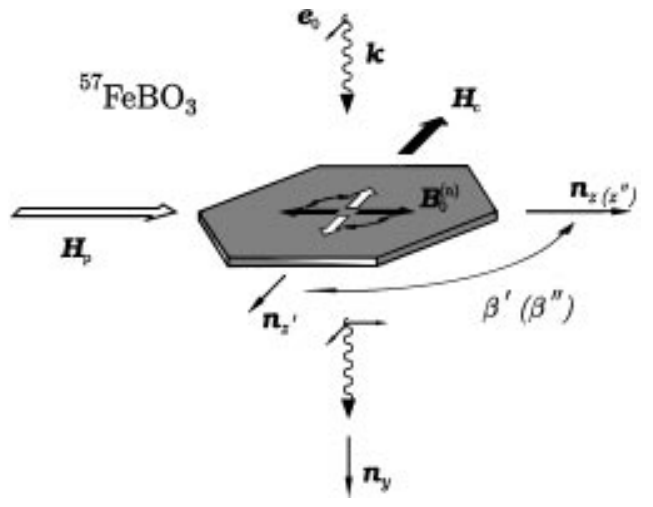

FIG. 1. Scattering geometry and scheme of switching the hyperfine field directions in the ${ }^{57} \mathrm{FeBO}_{3}$ crystal. The wave vector $\boldsymbol{k}$ indicates the incident radiation and radiation coherently reemitted in the forward direction. The polarization vector $\boldsymbol{e}_{0}$ of the electric field lies in the plane of the synchrotron orbit. Solid arrows $\boldsymbol{B}_{0}^{(n)}$ indicate the initial setting of the hyperfine fields at the two different sites $(n=1,2)$ in the crystal unit cell aligned by the external magnetic field $\boldsymbol{H}_{c}$. Open arrows indicate their setting after switching, which is performed at time $t^{\prime}$ by application of the external magnetic field $\boldsymbol{H}_{p} \perp \boldsymbol{H}_{c}$. The switching angle is denoted by $\beta^{\prime}$. Switching back by angle $\beta^{\prime \prime}=-\beta^{\prime}$ is achieved by turning off the field $\boldsymbol{H}_{p}$ at time $t^{\prime \prime}$. 


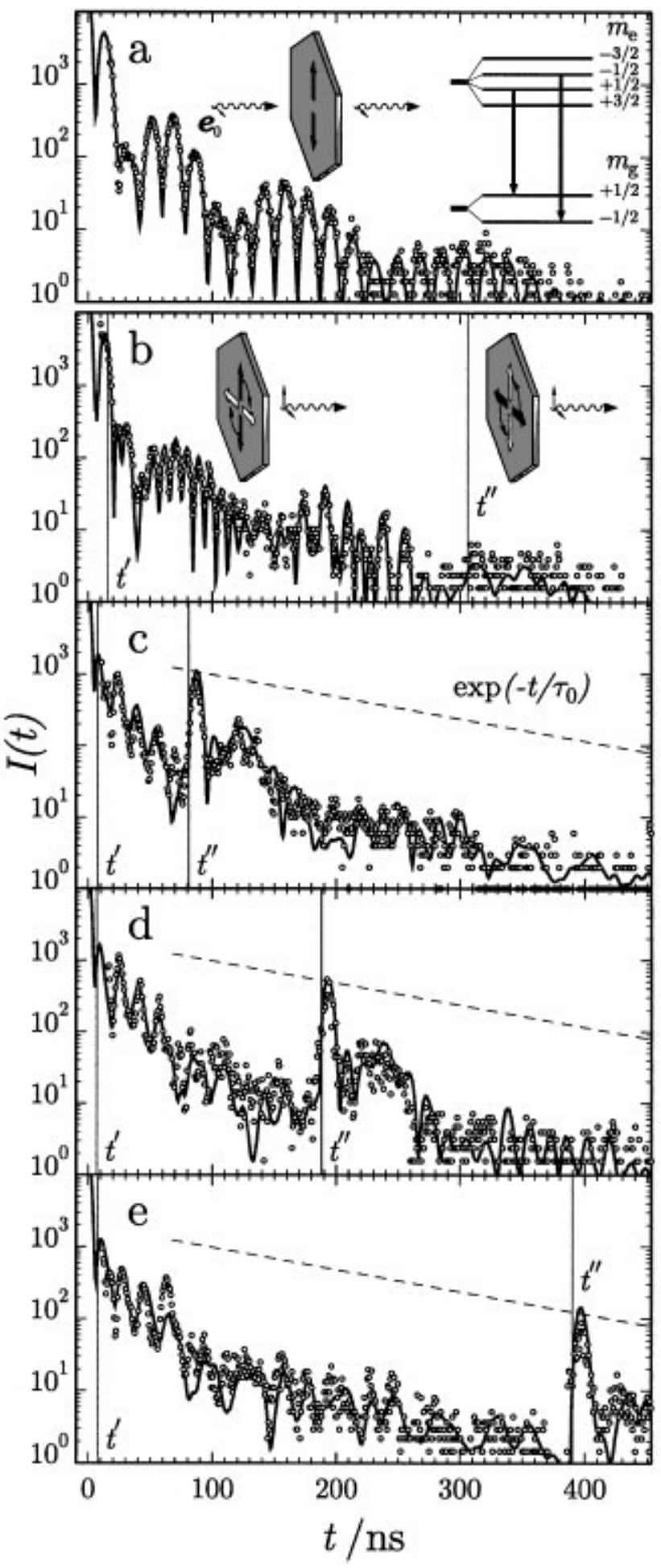

FIG. 2. Time spectra of the nuclear forward scattering in ${ }^{57} \mathrm{FeBO}_{3}$ : (a) unperturbed; (b) -(e) perturbed by switching the crystal magnetization forth at $t=t^{\prime}$ and back at $t=t^{\prime \prime}$ as indicated by the vertical lines. All spectra are normalized to equal incident integrated flux. The solid lines are the theoretical fits assuming instantaneous switching at $t^{\prime}, t^{\prime \prime}$. The best consistent fit of the data was obtained with a switching angle $\beta^{\prime}=-\beta^{\prime \prime}=72^{\circ}$. The dotted line indicates the natural decay. $n=1,2$ in the crystal unit cell in such a way that the incident beam in the $\boldsymbol{e}_{0}$ polarization state excited only the two $\Delta m=m_{g}-m_{e}=0$ transitions; see inset in Fig. 2(a). The measured time spectrum of the radiation intensity $I(t)$ reemitted into the forward direction shows the quantum beat pattern characteristic for nuclear scattering via these two transitions [11] [see Fig. 2(a)].

The application of a pulsed magnetic field $H_{p}=$ $58 \mathrm{G}$ in the crystal plane perpendicular to $H_{c}$ at time $t^{\prime}$ after the excitation [12] produces an abrupt rotation of the magnetization resulting in drastic changes in the time spectrum. The changes are very sensitive to $t^{\prime}$ : (i) Switching at $t^{\prime}=16 \mathrm{~ns}$, a time coinciding with a maximum in the unperturbed quantum beat pattern [Fig. 2(a)], results in the quite different time spectrum of Fig. 2(b), which corresponds to the emission, where only the $\Delta m= \pm 1$ transitions are involved. No change in the time-integrated count rate is observed. (ii) Switching at $t^{\prime}=8 \mathrm{~ns}$ [see Figs. 2(c)-2(e)], a time coinciding with the first minimum in the unpertured quantum beat pattern [Fig. 2(a)], preserves the $\Delta m=0$ pattern, but leads to a drastic reduction of the total number of counts $\mathcal{N}\left(t^{\prime}, t^{\prime \prime}\right)$ in the interval between $t^{\prime}$ and $t^{\prime \prime}$. The reduction is given by $\mathcal{N}_{x}\left(t^{\prime}, t^{\prime \prime}\right) / \mathcal{N}_{a}\left(t^{\prime}, t^{\prime \prime}\right) \approx 0.4 ; x=c, d, e$.

Switching back by turning off the field $H_{p}$ at $t^{\prime \prime}=81$, 188, and 390 ns [see Figs. 2(c)-2(e)] causes pronounced bursts of radiation in the forward direction. The total number of counts $\mathcal{N}_{x}\left(t^{\prime \prime}, \infty\right)$ after the second switching exceeds significantly the corresponding number of counts $\mathcal{N}_{a}\left(t^{\prime \prime}, \infty\right)$ in the unperturbed spectrum, e.g., $\mathcal{N}_{e}\left(t^{\prime \prime}, \infty\right) / \mathcal{N}_{a}\left(t^{\prime \prime}, \infty\right) \approx 75$. The time dependence of $\mathcal{N}_{x}\left(t^{\prime \prime}, \infty\right)$ can be described by the empirical law

$$
\begin{aligned}
\mathcal{N}_{x}\left(t^{\prime \prime}, \infty\right)= & {\left[\mathcal{N}_{a}\left(t^{\prime}, t^{\prime \prime}\right)-\mathcal{N}_{x}\left(t^{\prime}, t^{\prime \prime}\right)\right] } \\
& \left.\times \exp \left\{-t^{\prime \prime}-t^{\prime}\right) / \tau_{0}\right\} .
\end{aligned}
$$

Also the peak count rates of the radiation bursts obey a natural decay law as indicated by the guideline in Figs. 2(c)2(e). This indicates that the part of the excitation energy, the coherent reemission of which is forbidden as a result of the first switching, only decays with the natural lifetime $\tau_{0}$. Its decay is not speeded up, and this provides the possibility to store excitation energy.

To account for the changes in nuclear radiation caused by switching and to evaluate the time spectra the theory of the nuclear forward scattering in the presence of time-dependent hyperfine interactions has been developed $[13,14]$.

An ensemble of resonant nuclei with energy $E_{\lambda}$, spin $\boldsymbol{I}_{\lambda}$, magnetic moment $\mu_{\lambda}$ in the ground $(\lambda=g)$, and excited $(\lambda=e)$ states is considered. The nuclei are embedded in a crystal plate of thickness $L$ in the $y$ direction. A $\gamma$-radiation pulse of an amplitude $\boldsymbol{E}(0, t)=\epsilon \delta(t) \boldsymbol{e}_{0}$ at the crystal entrance surface $y=0$ excites the nuclear transitions $g \rightarrow e$ with energy $E_{0}=E_{e}-E_{g}$ and full natural width $\Gamma_{0}$. The amplitude $\boldsymbol{E}(L, t)$ of the radiation 
pulse caused by the coherent multiple forward scattering from the nuclei is calculated by the wave equation [14]

$$
\frac{d \boldsymbol{E}(y, t)}{d y}=-\sum_{\ell} K_{\ell} \boldsymbol{J}_{\ell}(t) \int_{-\infty}^{t} d \tau \boldsymbol{J}_{\ell}^{\dagger}(\tau) \cdot \boldsymbol{E}(y, \tau) .
$$

In compliance with the two-stage character of the resonance scattering the nuclear transition currents $\boldsymbol{J}_{\ell}(t) \equiv$ $\boldsymbol{J}_{m_{g} m_{e}}^{(n)}(t)$ [15], which are the matrix elements of the operator $\quad \hat{\boldsymbol{J}}^{(n)}(t)=\hat{U}_{\lambda}^{(n) \dagger}(t, 0) \hat{\boldsymbol{j}}(\boldsymbol{k}) \hat{U}_{\lambda}^{(n)}(t, 0) \exp \left(i E_{0} t / \hbar\right)$ between the ground and excited nuclear states $\left|m_{\lambda}\right\rangle$, occur twice in the wave equation: once due to the nuclear excitation at time $\tau$ and once due to the deexcitation at $t ; \hat{\boldsymbol{j}}(\boldsymbol{k})$ is the nuclear current density vector operator in momentum representation; $\hat{U}_{\lambda}^{(n)}\left(t_{2}, t_{1}\right)=$ $\hat{T} \exp \left[-\frac{i}{\hbar} \int_{t_{1}}^{t_{2}} d \tau \hat{\mathcal{H}}_{\lambda}^{(n)}(\tau)\right]$ and $\hat{T}$ are the evolution and the time-ordering operators, respectively; $\hat{\mathcal{H}}_{\lambda}^{(n)}(t)$ is the nuclear Hamiltonian; $K_{\ell}=2 \pi N_{n} f_{n}(\boldsymbol{k}) / k c^{2}\left(2 I_{g}+1\right)$, with $N_{n}$ being the number of the nuclear sites $n$ in the unit volume, and $f_{n}(\boldsymbol{k})$ being the Mössbauer factor.

The solution of Eq. (1) is given by the sum $\boldsymbol{E}(y, t)=$ $\boldsymbol{\epsilon} \sum_{n=0}^{\infty} \boldsymbol{E}_{n}(y, t)$ with $\boldsymbol{E}_{0}(y, t)=\delta(t) \boldsymbol{e}_{0}$ representing the prompt response. The other terms, e.g.,

$$
\boldsymbol{E}_{1}(y, t)=-y \sum_{\ell} K_{\ell} \boldsymbol{J}_{\ell}(t)\left[\boldsymbol{J}_{\ell}^{\dagger}(0) \cdot \boldsymbol{e}_{0}\right],
$$

representing delayed nuclear reemission are generated by iteration. The terms with $n=0$ and $n=1$ give the solution in the thin crystal limit which will be used in further discussion.

According to their definition $\boldsymbol{J}_{\ell}(t)$ are determined by the nuclear Hamiltonian, which in case of switching the magnetic hyperfine fields is equal to [16]

$$
\hat{\mathcal{H}}_{\lambda}^{(n)}(t)=E_{\lambda}-i \frac{\Gamma_{0}}{2} \delta_{\lambda, e}-\mu_{\lambda} \frac{\hat{\boldsymbol{I}}_{\lambda} \boldsymbol{B}^{(n)}(t)}{I_{\lambda}} .
$$

Initially, before switching $\left(0<t<t^{\prime}\right)$, the magnetic fields are constant $\boldsymbol{B}^{(n)}(t)=\boldsymbol{B}_{0}^{(n)}$ and aligned with $\boldsymbol{n}_{z}$. Under these conditions the transition currents are given by

$$
\boldsymbol{J}_{\ell}(t)=\boldsymbol{j}_{\ell}(\boldsymbol{k}) \exp \left[-i \Omega_{\ell} t\right] \zeta(t),
$$

where $\zeta(t)=\exp \left(-\Gamma_{0} t / 2 \hbar\right)$, and $\hbar \Omega_{\ell}=\left(\mu_{g} m_{g} / I_{g}-\right.$ $\left.\mu_{e} m_{e} / I_{e}\right) B_{0}^{(n)}$ is the correction to the transition energy $E_{0}$ due to the hyperfine interaction.

At $t=t^{\prime}$ the directions of the hyperfine fields are switched instantaneously to $\boldsymbol{n}_{z^{\prime}}$. This is described by $\boldsymbol{B}^{(n)}(t)=B_{0}^{(n)}\left[\boldsymbol{n}_{z} \theta\left(t^{\prime}-t\right)+\boldsymbol{n}_{z^{\prime}} \theta\left(t-t^{\prime}\right)\right]$ with $\theta(t)$ being the unit step

function and leads to

$$
\begin{aligned}
& \boldsymbol{J}_{\ell}(t)=\zeta(t) \sum_{\ell^{\prime}} \boldsymbol{j}_{\ell^{\prime}}(\boldsymbol{k}) \exp \left[-i \Omega_{\ell^{\prime}}\left(t-t^{\prime}\right)\right] S_{\ell^{\prime} \ell}^{\prime}, \\
& S_{\ell^{\prime} \ell}^{\prime}\left(\beta^{\prime}, t^{\prime}\right)=d_{m_{g}^{\prime} m_{g}}^{\left(I_{g}\right)}\left(\beta^{\prime}\right) d_{m_{e}^{\prime} m_{e}}^{\left(I_{e}\right)}\left(\beta^{\prime}\right) \exp \left[-i \Omega_{\ell} t^{\prime}\right] .
\end{aligned}
$$

Here $\beta^{\prime}$ is the switching angle, $d_{m_{\lambda}^{\prime} m_{\lambda}}^{\left(I_{\lambda}\right)}$ are the rotation matrices, and $m_{\lambda}^{\prime}$ are the spin projections on $\boldsymbol{n}_{z^{\prime}}$.

At $t=t^{\prime \prime}$ the magnetic hyperfine fields are switched to $\boldsymbol{n}_{z^{\prime \prime}}$. In the present experiment $\boldsymbol{n}_{z^{\prime \prime}}=\boldsymbol{n}_{z}$. The $\boldsymbol{J}_{\ell}(t)$ at $t>t^{\prime \prime}$ are described by the same Eq. (5), however, with the substitution $\ell^{\prime} \rightarrow \ell^{\prime \prime}$ and $S_{\ell^{\prime} \ell}^{\prime}\left(\beta^{\prime}, t^{\prime}\right) \rightarrow S_{\ell^{\prime \prime} \ell}^{\prime \prime}\left(\beta^{\prime \prime}, t^{\prime \prime}\right)$, where $S_{\ell^{\prime \prime} \ell}^{\prime \prime}$ is defined as

$$
S_{\ell^{\prime \prime} \ell}^{\prime \prime}\left(\beta^{\prime \prime}, t^{\prime \prime}\right)=\sum_{\ell^{\prime}} S_{\ell^{\prime \prime} \ell^{\prime}}^{\prime}\left(\beta^{\prime \prime}, t^{\prime \prime}-t^{\prime}\right) S_{\ell^{\prime} \ell}^{\prime}\left(\beta^{\prime}, t^{\prime}\right) .
$$

The theory outlined was used to evaluate unperturbed and perturbed time spectra $I(t) \propto|\boldsymbol{E}(L, t)|^{2}$; see solid lines in Fig. 2. It will be used as well in the following discussion of the changes in the time spectra noted above.

The incident radiation pulse excites monochromatic nuclear transitions $\ell$ [see Eq. (4)]. This results for the delayed spectrum [see Eq. (2)] in a sum of monochromatic components

$$
\boldsymbol{E}_{1}(L, t) \propto L \zeta(t) \sum_{\ell} \mathcal{A}_{\ell}(\boldsymbol{k}) \exp \left[-i \Omega_{\ell} t\right],
$$

with the amplitudes $\mathcal{A}_{\ell}(\boldsymbol{k})=\boldsymbol{j}_{\ell}(\boldsymbol{k}) K_{\ell}\left[\boldsymbol{j}_{\ell}^{\dagger}(\boldsymbol{k}) \cdot \boldsymbol{e}_{0}\right]$.

The switching transforms each initially excited monochromatic transition $\ell$ into a multiplet composed of all allowed transitions $\ell^{\prime}$ projected onto the new quantum axis $\boldsymbol{n}_{z^{\prime}}$ [see Eq. (5)]. Despite this complication, the time spectrum is given by the same Eq. (8), however, with the substitution $\ell \rightarrow \ell^{\prime}, t \rightarrow\left(t-t^{\prime}\right)$ on the right-hand side. The amplitudes are now built up by the interference of the initially excited transitions $\ell$ : $\mathcal{A}_{\ell^{\prime}}(\boldsymbol{k})=\boldsymbol{j}_{\ell^{\prime}}(\boldsymbol{k}) \sum_{\ell} S_{\ell^{\prime} \ell}^{\prime}\left(\beta^{\prime}, t^{\prime}\right)\left[\boldsymbol{j}_{\ell}^{\dagger}(\boldsymbol{k}) \cdot \boldsymbol{e}_{0}\right]$. Depending on the values of $\beta^{\prime}$ and $t^{\prime}$, the interference may result in the creation of new frequency components as well as in the suppression of already excited ones. It may even happen that all the components and thus the total coherent reemission are suppressed. However, this implies by no means that the transition currents $\boldsymbol{J}_{\ell}(t)$ are annihilated. Virtually they still exist. Therefore one can change the interference by the next switching and thus recover the coherent emission.

For the conditions of our experiment this general description gives the following picture. For simplicity we shall discuss $90^{\circ}$ switching. The doublet of $\Delta m=0$ transitions with the energies $E_{0} \pm \hbar \Omega$ is excited; see Fig. 3(a). This results in a time spectrum given by $\boldsymbol{E}(t) \propto 2 \zeta(t) \mathcal{A}(\boldsymbol{k}) \cos \Omega t$ [see Eq. (8)] with $\mathcal{A}(\boldsymbol{k}) \propto \boldsymbol{k} \times \boldsymbol{n}_{z}$ [17]. The switching splits each excited monochromatic transition into sextets. It is important that in case of the $90^{\circ}$ switching the components having the same energies are of equal strength in both sextets [13]. They differ only by their signs and time phases; see Fig. 3(b). In the time spectrum the interference of the sextets leads to amplitudes $\mathcal{A}_{\ell^{\prime}}(\boldsymbol{k}) \propto \frac{1}{2} \boldsymbol{k} \times$ $\boldsymbol{n}_{z^{\prime}} \sin \Omega t^{\prime}$ for the $\Delta m^{\prime}=0$ and $\mathcal{A}_{\ell^{\prime}}(\boldsymbol{k}) \propto \boldsymbol{n}_{\mathbf{z}^{\prime}} \cos \Omega t^{\prime}$ 


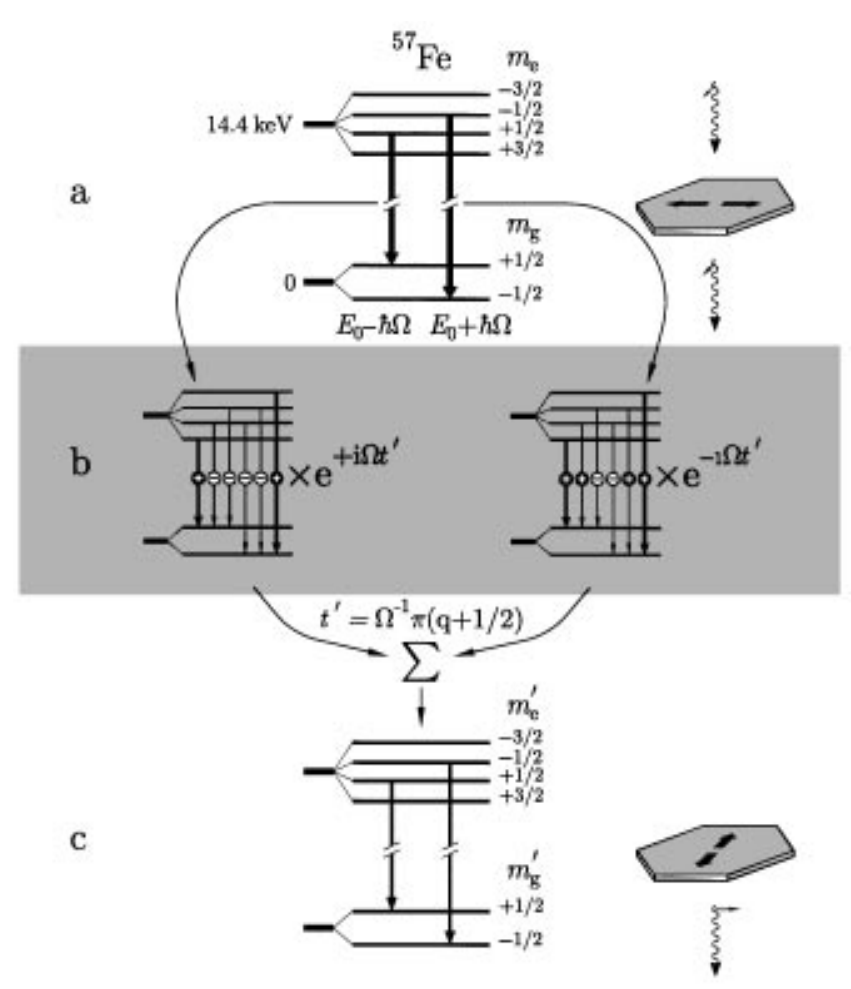

FIG. 3. Scheme of transformations in the radiation of ${ }^{57} \mathrm{Fe}$ nuclei caused by $90^{\circ}$ switching. (a) Before switching. Decay via the transitions $\Delta m=0$ excited by synchrotron radiation. (b) Switching transforms each monochromatic transition into a sextet. The strength of similar transitions in the two sextets are equal. Their phases are different and are determined by the signs of the elements of the rotation matrices and by $\exp \left( \pm i \Omega t^{\prime}\right)$ originating from the parent transitions. If, e.g., $t^{\prime}=\Omega^{-1} \pi\left(q+\frac{1}{2}\right), q=0,1, \ldots$, a pure $\Delta m^{\prime}=0$ spectrum results (c).

for the $\Delta m^{\prime} \pm 1$ components. In agreement with experiment, switching at $t^{\prime}=\Omega^{-1} \pi q, q=0,1, \ldots$, i.e., at times which coincide with a maximum in the unperturbed time spectrum, results in radiation comprising only the $\Delta m^{\prime}= \pm 1$ components. Switching in a minimum, i.e., at $t^{\prime}=\Omega^{-1} \pi\left(q+\frac{1}{2}\right)$, results in radiation comprising the same $\Delta m^{\prime}=0$ components as in the unperturbed spectrum, however, with their amplitudes reduced by a factor of 2 and in a different polarization state; see Fig. 3(c).

We want to emphasize that switching the magnetic hyperfine field directions into a direction $\boldsymbol{n}_{z^{\prime}}$ parallel to $\boldsymbol{k}$ would result in a complete suppression of the coherent emission. Nevertheless, switching back changes again the interference of the transitions and should restore strong reemission into the forward direction. Experiments into this direction are in preparation.

In summary, we have demonstrated the possibility to control the total coherent decay rate and to store nuclear excitation energy by magnetic switching. The experimental results are well described by the theory.
Suppression and restoration of the coherent nuclear decay originate from drastic changes of the nuclear states and of the interference within the nuclear transitions caused by the magnetic switching.

This work has been funded by the Bundesministerium für Bildung, Wissenschaft, Forschung und Technologie under Contracts No. 05 643GUA1 and No. 05 5WOAAI/643WOA.

[1] Yu. V. Shvyd'ko, A.I. Chumakov, G. V. Smirnov, T. Hertrich, U. van Bürck, H.D. Rüter, O. Leupold, J. Metge, and E. Gerdau, Europhys. Lett. 26, 215 (1994).

[2] G. T. Trammell, in Chemical Effects of Nuclear Transformations (IAEA, Vienna, 1961), Vol. I, p. 75.

[3] A. M. Afanas'ev and Yu. Kagan, Pis'ma Zh. Eksp. Teor. Fiz. 2, 130 (1965) [JETP Lett. 2, 81 (1965)].

[4] J. P. Hannon and G. T. Trammell, in Resonant Anomalous $X$-ray Scattering, edited by G. Materlik, C. J. Sparks, and K. Fischer (North-Holland, Amsterdam, 1994), p. 565.

[5] R. H. Dicke, Phys. Rev. 93, 99 (1954).

[6] J.E. Rothenberg, D. Grischkovsky, and A.C. Balant, Phys. Rev. Lett. 53, 552 (1984).

[7] D. Fröhlich, A. Kulik, B. Uebbing, A. Mysyrowicz,, V. Langer, H. Stolz, and W. von der Osten, Phys. Rev. Lett. 67, 2343 (1991).

[8] R. G. DeVoe and R. G. Brewer, Phys. Rev. Lett. 76, 2049 (1996).

[9] R. Rüffer, D. Giesenberg, H. D. Rüter, R. Hollatz, E. Gerdau, J. Metge, K. Ruth, W. Sturhahn, M. Grote, and R. Röhlsberger, Hyperfine Interact. 58, 2467 (1990).

[10] The high resolution monochromator with channel-cut $\mathrm{Si}(4$ 2 2) and $\operatorname{Si}\left(\begin{array}{lll}12 & 2 & 2\end{array}\right)$ crystals in the nested geometry was designed by E. Gerdau, R. Rüffer, and H. D. Rüter based on the proposal of T. Ishikawa, Y. Yoda, K. Izumi, C. K. Suzuki, X. W. Zhang, M. Ando, and S. Kikuta, Rev. Sci. Instrum. 63, 1015 (1992).

[11] U. van Bürck, D. P. Siddons, J. B. Hastings, U. Bergmann, and R. Hollatz, Phys. Rev. B 46, 6207 (1992).

[12] For more details on the measurement procedure, see, e.g., [1]; Yu. V. Shvyd'ko, T. Hertich, J. Metge, O. Leupold, E. Gerdau, and H. D. Rüter, Phys. Rev. B 52, R711 (1995); Yu. V. Shvyd'ko, S. L. Popov, and G. V. Smirnov, J. Phys. Condens. Matter 5, 1557 (1993).

[13] Yu. V. Shvyd'ko, Hyperfine Interact. 90, 287 (1994).

[14] Yu. V. Shvyd'ko (to be published).

[15] Unless it causes ambiguities a joint index $\ell \equiv\left\{m_{g} \rightarrow\right.$ $\left.m_{e},(n)\right\}$ is used for brevity to denote both the nuclear transition and the site numbers.

[16] ${ }^{57} \mathrm{Fe}$ nuclei in ${ }^{57} \mathrm{FeBO}_{3}$ experience both magnetic and a weak quadrupole hyperfine interaction. This was rigorously taken into account in the evaluation of the time spectra shown in Fig. 2. However, for more vividness we omit in the discussion outlined in this Letter the influence of the quadrupole interaction.

[17] Here the explicit expression for the $\boldsymbol{j}_{m_{g} m_{e}}(\boldsymbol{k})$ for the $M 1$ nuclear transitions was used. See, e.g., Yu. M. Kagan and A. M. Afanas'ev, Z. Naturforsch. 28a, 1351 (1973). 